


\title{
Geschichte der Chemie
} von den ältesten Zeiten bis zur Gegenwart.

Zugleich Einführung in das Studium der Chemie.

$$
\text { Von }
$$

Dr. Ernst von Meyer,

Gel. Hofrat, o. Professor der Chemie an der Technischen Hochschule zu Dresden.

Vierte, verbesserte und vermehrte Auflage.

gr. 8. geh. $13 \mathcal{k}$, geb. in Ganzleinen 14

\section{Praktikum des anorganischen Chemikers.}

Einführung in die anorganische Chemie auf experimenteller Grundlage

\author{
von
}

Dr. Emil Knoevenagel,

Professor an der Universität Heidelberg.

Zweite, vollständig veränderte Auflage, mitbearbeitet von

Professor Dr. Erich Ebler in Heidelberg.

Mit zahlreichen Figuren, vier Tabellen und neun Tafeln.

gr. 8. geb. in Ganzleinen $10 \mathrm{Ab}$.

\section{Die}

\section{Mineral-, Pflanzen- und Teerfarben.}

Ihre Darstellung, Verwendung, Erkennung und Echtheitsprüfung.

$$
\text { Von }
$$

Dr. Hans Th. Bucherer, Professor an der 'Technischen Hochschule zu Dresten.

$$
\text { Mit } 4 \text { Tafeln. }
$$

gr. 8. geh. $3 \stackrel{16}{60} 9 \%$

\section{Quantitative Elektroanalyse.}

Mit einem Anhang:

Organische Elementaranalyse auf elektrischem Wege.

$$
\text { Von }
$$

Dr. Edgar F. Smith,

Professor der Chenie an der Pennsylvania-Universität zu Philadelphia.

Nach der vierten Auflage mit Genehmigung des Verfassers deutsch bearbeite't von

Dr. Arthur Stähler,

Privatdozent der Chemie an der Universität Berlin.

Mit 43 Figuren.

gr. 8. geb. in Ganzleinen $8 \mathcal{H}$. 


\section{Die Praxis des organischen Chemikers.}

$$
\text { Von }
$$

Dr. phil. Ludwig Gattermann,

o. Professor der ('hemio und Direktor der naturwissenschaftllch-mathematischen Abteilung des chemischen Laboratoriums der Universität Freiburg i. B.

Zwölfte, verbesserte Auflage.

Mit 95 Abbildungen und zwei Tabellen.

gr. 8. geb. in Ganzleinen 8 H6 50 gF.

\section{Terpene und Campher.}

Zusammenfassung eigener Untersuchungen auf dem Gebiete

der alicyclischen Kohlenstoffverbindungen

$$
\text { von }
$$

Dr. Otto Wallach,

Geh. Reg.-Rat, o. ö. Professor der Chemie an der Universităt Göttingen.

$\mathrm{Zweite}$ Auflage.

Lex. 8. geh. $24 \mathscr{H}$, geb. in Halbfranz $27 \stackrel{\mathscr{H}}{ }$.

\section{Praktischer \\ Leitfaden der Gewichtsanalyse. Von}

Dr. Paul Jannasch,

Professor an der Universität Heidelberg.

Zweite, vermehrte und verbesserte Auflage.

Mit zahlreichen Abbildungen im Text.

gr. 8. geb. in Ganzleinen $8 \mathscr{K}$.

\section{Nahrungsmittelchemie und Nahrungsmittelkontrolle} in ihrer Bedeutung für Handel, Gewerbe

\section{und Industrie.}

Mit den einschlägigen Gesetzen und Verordnungen.

$$
\text { Von }
$$

\section{Dr. A. Kraus,}

Kreischemiker und Vorsteher des Nahrungsmittel-Untersuchungsantes in Neuß a. Rh.

8. geb. in Ganzleinen $3 \mathscr{A 6} 50$ gF.

\section{Die ätherischen Öle}

nach ihren chemischen Bestandteilen

unter Berücksichtigung der geschichtlichen Entwicklung.

$$
\text { Von }
$$

Dr. F. W. Semmler,

Professor der Chemie an der Tecbnischen IJochschule Breslau.

Vier Bände in Royal-Oktavformat.

geh. 132 /6, geb. in Halbfranz 146 $/ 6$. 


\section{Lehrbuch der organischen Chemie}

für Studierende an Universitäten und Technischen Hochschulen.

$$
\text { Von }
$$

Dr. A. F. Holleman,

o. Professor der Chemie an der Unlversität Amsterdam.

Zwölfte, verbesserte Auflage. Mit zahlreichen Figuren.

gr. 8. geb. in Ganzleinen $10 \mathrm{H}$.

\section{Die direkte Einführung von Substituenten in den Benzolkern.}

Fin Beitrag zur Lösung des Substitutionsproblems in aromatischen Verbindungen.

Kritische Literaturübersicht und experimentelle Untersuchungen.

$$
\text { Vou }
$$

Dr. A. F. Holleman.

Mit zahlreichen Figuren.

Lex. 8. geh. $20 ~ \mathfrak{H}$, geb. in Halbfranz $23 \ldots$.

\section{Einfache Versuche auf dem Gebiete der organischen Chemie.}

Eine Anleitung für Studierende, Lehrer an höheren

Schulen und Seminaren, sowie zum Selbstunterricht.

$$
\text { Von }
$$

Dr. A. F. Holleman.

Zweite, verbesserte Auflage. Mit Figuren.

1916. 8. gebunden in Ganzleinen $2 \mathscr{H} 60$ gg.

\section{Refraktometrisches Hilfsbuch.}

\section{Von}

Dr. W. A. Roth und Dr. F. Eisenlohr

a. o. Professor

Privatdozent

an der Universität Greifswald.

Mit 27 Figuren, 17 Tabellen, sowie Logarithmen.

gr. 8. geb. in Ganzleinen 6 .

Ein neuartiges Laboratoriums-Hilfsbuch, durch das ein längst empfundenes dringendes Bedürfnis befriedigt wird.

Nicht nur aus rein physikalisch-chemischen Gründen, sondern namentlich zum "Wwecke der Konstitutionsbestimmung organischer Verbindungen befaBt sich gerade in neuerer Zeit eine groBe Anzahl von Chemikern mit der Bestimmung von Molekularrefraktionen und -dispersionen, wobei wegen Mangels an geeigneten Tabellen höchst mühsame und unbequeme Berechnungen erforderlich sind.

Dieser große Übelstand wird durch das neue „Refraktometrische Hilfsbuch" von Roth und Eisenlohr mit einem Schlage beseitigt. 



\title{
LEHRBUCH
}

\section{DER \\ C H E M I E}

VON

\author{
DR. A. F. HOLLEMAN, \\ O. PROPEsBor DER CHEMIE AN DER UNIVERBITÄT AMSTERDAM.
}

AUTORISIERTE DEUTSCHE AUSGABE.

ANORGANISCHER TEIL.

DREIZEHNTE, VERBESSERTE AUFLAGE.

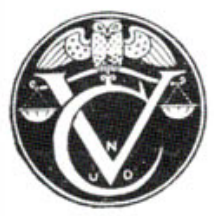

LEIPZIG

VERLAG VON VEIT \& COMP. 


\section{LEHRBUCH}

\section{DER \\ ANORGANISCHEN CHEMIE}

FÜR STUDIERENDE

AN UNIVERSITÄTEN UND TECHNISCHEN HOCHSCHULEN

voN

DR. A. F. HOLLEMAN,

o. PROFESSOR DER CHEMIE AN DER UNIVERgITÄT AMSTERDAM.

DREIZEHNTE, VERBESSERTE AUFLAGE.

MIT ZAHLREICHEN FIGUREN, EINER TABELLE UND ZWEI TAFELN.

LEIPZIG

VERLAG VON VEIT \& COMP. 
Die Elnfuhrang dieser deutachen Originnlausgabe nsch Holland ist untersagt. 